\title{
Condition for macroscopic realism beyond the Leggett-Garg inequalities
}

\author{
Johannes Kofler ${ }^{1}$ and Časlav Brukner ${ }^{2,3}$ \\ ${ }^{1}$ Max Planck Institute of Quantum Optics (MPQ), Hans-Kopfermann-Straße 1, 85748 Garching/Munich, Germany \\ ${ }^{2}$ Faculty of Physics, University of Vienna, Boltzmanngasse 5, 1090 Vienna, Austria \\ ${ }^{3}$ Institute for Quantum Optics and Quantum Information, \\ Austrian Academy of Sciences, Boltzmanngasse 3, 1090 Vienna, Austria
}

(Dated: May 21, 2018)

\begin{abstract}
In 1985, Leggett and Garg put forward the concept of macroscopic realism (macrorealism) and, in analogy to Bell's theorem, derived a necessary condition in terms of inequalities, which are now known as the Leggett-Garg inequalities. In this paper, we discuss another necessary condition called no-signaling in time. It solely bases on comparing the probability distribution for a macrovariable at some time for the cases where previously a measurement has or has not been performed. Although the concept is analogous to the no-signaling condition in the case of Bell tests, it can be violated according to quantum mechanical predictions even in situations where no violation of Leggett-Garg inequalities is possible.
\end{abstract}

Bell's theorem for local realism [1] is a highly developed research field, not least because of its importance for quantum information technologies [2]. Macroscopic realism (macrorealism) [3] - the world view in which the properties of macroscopic objects exist independent of and are not influenced by measurement-has gained momentum only within the past few years as experiments steadily approached the parameter regime where experimental tests might become possible. Promising candidates in the race towards an experimental violation of macrorealism are large superconducting devices [4, 5], heavy molecules [6, 7], and quantum-optical systems in combination with atomic gases [8] or massive objects [9]. Still lacking a decisive experiment, however, the physics community remains to be split into two groups: adherents of the viewpoint that macrorealism will eventually be falsified by the preparation of Schrödinger cat-like states [10], and adherents of one of the hypothetical alternatives saving a classical world on the macroscopic level [11-13].

Macrorealism is defined by the following postulates [14]: "(1) Macrorealism per se. A macroscopic object which has available to it two or more macroscopically distinct states is at any given time in a definite one of those states. (2) Noninvasive measurability. It is possible in principle to determine which of these states the system is in without any effect on the state itself or on the subsequent system dynamics. (3) Induction. The properties of ensembles are determined exclusively by initial conditions (and in particular not by final conditions)."

Since an observation of quantum interference between macroscopically distinct states (QIMDS), as predicted by quantum mechanics $(\mathrm{QM})$, does not necessarily establish the falsity of macrorealism, three stages of experiments should be distinguished [14]: "Stage 1. One conducts circumstantial tests to check whether the relevant macroscopic variable appears to be obeying the prescriptions of QM. Stage 2. One looks for direct evidence for QIMDS, in contexts where it does not (necessarily) exclude macrorealism. Stage 3. One conducts an experiment which is explicitly designed so that if the results specified by QM are observed, macrorealism is thereby excluded." Leggett and Garg have put forward the structure of such a stage 3 experiment [3]. It consists of measuring temporal correlation functions and violation of the so called Leggett-Garg inequalities.

In this work, we derive a necessary mathematical condition for macrorealism alternative to the Leggett-Garg inequalities, which we call no-signaling in time. A similar version of this condition was already discussed in Refs. [15-17] and independently found in Ref. [18] in the context of coarse-grained measurements of large spin systems. In Ref. [19] the strength of signaling in a temporal Clauser-Horne-Shimony-Holt scenario was discussed. However, in none of these references was it recognized as being experimentally more applicable than the Leggett-Garg inequalities. The condition bases solely on the time evolution of the probability distribution associated with a macroscopic quantity and can be viewed as a statistical version of non-invasive measurability. Only two measurement times are required, while any Leggett-Garg inequality necessarily involves at least three of them, making a conclusive test of macrorealism more feasible. We will apply the no-signaling in time condition to the specific case of interferometric experiments. Once quantum interference between macroscopically distinct states is shown, it suffices to demonstrate that it disappears when a prior measurement is made. Our work thus suggests that the step from a stage 2 to a stage 3 experiment usually can be done in a straightforward way. We start our analysis with a comparison between local realism and macrorealism.

Two parties, Alice and Bob, perform measurements on distant particles. Alice's (Bob's) setting choices are labeled with $a=a_{1}, a_{2}, \ldots\left(b=b_{1}, b_{2}, \ldots\right)$, and her (his) outcomes for a given setting are denoted by $A(B)$. The assumptions for local realism can be formulated as follows: Realism is a worldview "according to which external reality is assumed to exist and have definite properties, whether or not they are observed by someone" [20]. Locality demands that "if two measurements are made at places remote from one another the [setting of one measurement device] does not influence the result obtained with the other" [1]. There is also a third assumption, namely the freedom of choosing the settings independently of the particle properties. The joint assumption is denoted as lo- 
cal realism (LR) and demands that the probability for obtaining outcomes $A$ and $B$ under settings $a$ and $b$ can be written as a convex combination of products of probabilities which depend only on the local setting and a shared (hidden) variable $\lambda$, which specifies the properties of every individual particle pair and is generated with some probability distribution $\rho(\lambda)$ [42]:

$$
\text { LR: } P(A, B \mid a, b)=\sum_{\lambda} \rho(\lambda) P(A \mid a, \lambda) P(B \mid b, \lambda) .
$$

A special case of local realism is local determinism, where the outcome probabilities $P(A \mid a, \lambda)$ and $P(B \mid b, \lambda)$ are always either 0 or 1 .

Quantum mechanics, on the other hand, defines measurement operators $\hat{M}_{A}^{a}$ and $\hat{M}_{B}^{b}$ for Alice's and Bob's outcomes $A$ and $B$ under settings $a$ and $b$, respectively. The outcome probability for a given (bipartite) quantum state $\hat{\rho}$ is

$$
\text { QM: } P(A, B \mid a, b)=\operatorname{Tr}\left[\hat{\rho} \hat{M}_{A}^{a} \otimes \hat{M}_{B}^{b}\right] .
$$

In a basic scenario, there are only two setting choices for each party, $a=a_{1}, a_{2}, b=b_{1}, b_{2}$, and dichotomic outcomes, $A_{a}= \pm 1, B_{b}= \pm 1$. According to Bell's theorem, local realism puts a bound on certain combinations of correlation functions $C_{a b}=\left\langle A_{a} B_{b}\right\rangle$ for distant measurements. This leads to Bell inequalities (BI), e.g. the version by Clauser, Horne, Shimony and Holt (CHSH) [21]:

$$
\text { BI: } C_{a_{1} b_{1}}+C_{a_{2} b_{1}}+C_{a_{2} b_{2}}-C_{a_{1} b_{2}} \leq 2 .
$$

Bell inequalities can be violated by entangled quantum states.

All theories in accordance with the principle of nosignaling (NS) have to ensure that the outcome probabilities for one party must not depend on the setting of the other party in case the relevant events are space-like separated:

$$
\text { NS: } P(B \mid b)=P(B \mid a, b)=\sum_{A} P(A, B \mid a, b),
$$

and vice versa for $P(A \mid a)$. Here, $\sum_{A} P(A, B \mid a, b)=$ $\sum_{A} P(A \mid a, b) P(B \mid A, a, b)$, and the sum is taken over all possible results $A$ [43].

Local realism implies both the Bell inequalities and the no-signaling condition. However, while all local realistic correlations are no-signaling, the opposite does not necessarily hold. For instance, quantum mechanical correlations or PR boxes [22] are no-signaling but violate local realism, which means that the correlations cannot be decomposed as in eq. (1). Thus, no-signaling does not allow to derive Bell's inequalities. As one cannot reasonably hope to disproove local realism by observing a violation of the no-signaling condition, it is indeed necessary to check multiple correlation functions and violate Bell's inequality.

Now we turn to macrorealism. We consider a macroscopic object which is described by a set of macrovariables $\left\{Q, Q^{\prime}, \ldots\right\}$, whose values are considered to be macroscopically distinct by some measure [14]. Examples are the coarsegrained position and momentum for heavy particles in phase space, the charge and trapped flux in a superconducting quantum interference device (SQUID), or the magnetic moment along different directions of large biomolecules. In a series of runs, the object is prepared in the same initial state, and each preparation defines a new origin of the time axis $t=0$. Let us consider the case where macrovariable $A \in\left\{Q, Q^{\prime}, \ldots\right\}$ is measured at time $t_{A}\left(t_{A}>0\right)$ and macrovariable $B \in\left\{Q, Q^{\prime}, \ldots\right\}$ at later time $t_{B}\left(t_{B}>t_{A}\right)$. (One may of course choose $A=B$ and measure the same observable twice.) The induction postulate is reflected by the freedom of choosing the measurement times independently of the properties of the initially prepared objects. In analogy with eq. (1), macrorealism predicts that the probability for observing the outcomes $A$ at $t_{A}$ and $B$ at $t_{B}$ can we written as a convex combination of products of probabilities where the later measurement outcome does not depend on the earlier measurement [44]:

$$
\text { MR: } P\left(A_{t_{A}}, B_{t_{B}}\right)=\sum_{\lambda} \rho(\lambda) P\left(A_{t_{A}} \mid \lambda\right) P\left(B_{t_{B}} \mid \lambda\right) .
$$

There are two possible ways to define $\lambda$. For every preparation, it can represent a complete catalogue specifying all properties $\left\{Q, Q^{\prime}, \ldots\right\}$ of the object either (i) for all times or (ii) only at the initial time. In case (i), given $\lambda$, the probabilities $P\left(A_{t_{A}} \mid \lambda\right)$ and $P\left(B_{t_{B}} \mid \lambda\right)$ have to be either 0 or 1 . This is due to the postulate that every macrovariable must always have a definite value. Stochastic time evolutions are taken into account by a non-trivial distribution $\rho(\lambda)$, allowing different $\lambda$ even for identically prepared objects. In case (ii), a complete description $\lambda_{t}$ of the object at later times $t$ is not determined by $\lambda$, if the time evolution is stochastic even when $\rho(\lambda)$ is non-zero only for one $\lambda$. The time evolution of $\lambda$ must not be influenced by the measurements [45].

In contrast to macrorealism, quantum mechanics predicts the outcome probability

$$
\text { QM: } P\left(A_{t_{A}}, B_{t_{B}}\right)=\operatorname{Tr}\left[\hat{\rho}\left(t_{A}\right) \hat{M}_{A}\right] \operatorname{Tr}\left[\hat{\rho}_{A_{t_{A}}}\left(t_{B}\right) \hat{M}_{B}\right] .
$$

Here, $\hat{\rho}\left(t_{A}\right)$ is the state at time $t_{A}, \hat{M}_{A}$ and $\hat{M}_{B}$ are the measurement operators for outcomes $A$ and $B$, and $\hat{\rho}_{A_{t_{A}}}\left(t_{B}\right)$ is the (reduced) quantum state at time $t_{B}$ given that at time $t_{A}$ result $A$ was obtained.

In the simplest case, a single macrovariable $Q$ may only obtain two different values $Q= \pm 1$. Macrorealism restricts the allowed temporal correlations $C_{t_{A} t_{B}} \equiv\left\langle Q_{t_{A}} Q_{t_{B}}\right\rangle$ for measurements at $t_{A}$ and $t_{B}$ and implies the Leggett-Garg inequalities (LGI) [3], e.g. of the CHSH type $\left(t_{1}<t_{2}<t_{3}<t_{4}\right)$ :

$$
\text { LGI: } C_{t_{1} t_{2}}+C_{t_{2} t_{3}}+C_{t_{3} t_{4}}-C_{t_{1} t_{4}} \leq 2 \text {. }
$$

There is a one-to-one correspondence with the CHSH version of Bell's inequality (3). Alice's and Bob's setting choices correspond to the measuring times of $Q$ in the following way: $a_{1} \leftrightarrow t_{1}, b_{1} \leftrightarrow t_{2}, a_{2} \leftrightarrow t_{3}, b_{2} \leftrightarrow t_{4}$. (For a discussion about "entanglement in time" see Ref. [23].)

A violation of the Leggett-Garg inequality is ubiquitous in the microscopic quantum world [18, 24--29]. An experimental demonstration of macrorealism with its reference to macroscopically distinct states is, as pointed out above, still missing. This is due to the fact that one needs to engineer time 
evolutions (Hamiltonians) which build up macroscopic superpositions in time [18] and to perform multiple temporal correlation measurements before the superpositions are destroyed by decoherence. Whenever one talks about macroscopic or classical measurements of quantum systems, one should have in mind coarse-grained measurements which bunch together those quantum levels to "reasonable" [30] observables that are neighboring in the sense of classical physics.

Based on Refs. [15-19] we now make the following definition: "No-signaling in time: A measurement does not change the outcome statistics of a later measurement." No-signaling in time (NSIT) is obeyed by all macrorealistic theories and demands that the probability for macrovariable $B$ at time $t_{B}$ without any earlier measurement, $P\left(B_{t_{B}}\right)$, must be the same as $P\left(B_{t_{B} \mid t_{A}}\right)$ where also an earlier measurement of an arbitrary macrovariable $A$ has been made at $t_{A}$ :

$$
\text { NSIT: } P\left(B_{t_{B}}\right)=P\left(B_{t_{B} \mid t_{A}}\right)=\sum_{A} P\left(A_{t_{A}}, B_{t_{B}}\right)
$$

Here, $\sum_{A} P\left(A_{t_{A}}, B_{t_{B}}\right)=\sum_{A} P\left(A_{t_{A}}\right) P\left(B_{t_{B}} \mid A_{t_{A}}\right), P\left(A_{t_{A}}\right)$ is the probability for result $A$ at $t_{A}$, and $P\left(B_{t_{B}} \mid A_{t_{A}}\right)$ is the probability for outcome $B$ at $t_{B}$, given result $A$ was obtained at $t_{A}$. If we denote the probability amplitudes for the results $A$ by $a_{A}\left(\left|a_{A}\right|^{2}=P\left(A_{t_{A}}\right)\right)$ and the transition probability amplitudes from $A$ to $B$ by $a_{A \rightarrow B}\left(\left|a_{A \rightarrow B}\right|^{2}=P\left(B_{t_{B}} \mid A_{t_{A}}\right)\right)$, then the difference between the left and right hand side of $(8)$ reads $P\left(B_{t_{B}}\right)-P\left(B_{t_{B} \mid t_{A}}\right)=\left|\sum_{A} a_{A} a_{A \rightarrow B}\right|^{2}-\sum_{A} P\left(A_{t_{A}}\right) P\left(B_{t_{B}} \mid A_{t_{A}}\right)$. This shows that the violation of no-signaling in time [46] is exactly given by the quantum mechanical interference terms [31].

No-signaling in time is the analog of the no-signaling condition (4) in the sense that both are operationally testable and can be viewed as statistical versions of non-invasive measurability and locality, respectively. The key difference is that a violation of no-signaling in time is not at variance with special relativity and can be achieved by quantum mechanics.

Macrorealism implies both the Leggett-Garg-inequalities and no-signaling in time. But the latter does in general not allow to derive Leggett-Garg inequalities, which can be seen from the following (thought) experiment: Consider an ensemble, where initially, at time $t=0$, half of the systems are in the state $Q=+1$ and the other half in $Q=-1$. Let the time evolution be the macroscopic analog of a precessing spin- $\frac{1}{2}$ particle with frequency $\omega[24]$. Macroscopic quantum superpositions are produced in time in such a way that the temporal correlation function for measurements at times $t_{A}$ and $t_{B}$ reads $C_{t_{A} t_{B}} \equiv$ $\left\langle Q_{t_{A}} Q_{t_{B}}\right\rangle=\cos \left[\omega\left(t_{B}-t_{A}\right)\right]$. Suitable measurement times allow for a (maximal) violation of the Leggett-Garg inequality (7), while no-signaling in time is still fulfilled between any pair of measurements: $P\left(Q_{t_{B}}=+1\right)=P\left(Q_{t_{B} \mid t_{A}}=+1\right)=\frac{1}{2}$. Due to the mixedness of the initial state, the violation of macrorealism can hide in the statistics of condition (8). However, if a given Hamiltonian permits to violate macrorealism, then any initial pure state allows one to find time instances $t_{A}$ and $t_{B}$ such that no-signaling in time can be violated. To show this, it is enough to notice that a violation of macrorealism requires interference of superposition branches. An intermediate mea-

\begin{tabular}{ll}
\hline \hline Local Realism (LR) & Macrorealism (MR) \\
\hline Bell inequality (BI) & Leggett-Garg inequality (LGI) \\
No-signaling (NS) & No-signaling in time (NSIT) \\
LR $\Rightarrow$ BI & MR $\Rightarrow$ LGI \\
LR $\Rightarrow$ NS & MR $\Rightarrow$ NSIT \\
NS $\nRightarrow$ BI & NSIT $\nRightarrow$ LGI \\
QM $\nRightarrow$ BI & QM $\nRightarrow$ LGI \\
$Q M \Rightarrow N S$ & $Q M \nRightarrow N S I T$ \\
\hline \hline
\end{tabular}

TABLE I: Local realism and macrorealism are largely analogous in their conceptual relationships. The Bell and Leggett-Garg inequalities are both violated by quantum mechanics (QM). The key difference (written in italics) is that quantum mechanics obeys nosignaling while it can violate no-signaling in time.

surement destroys the interference term and thus makes itself detectable at a later time.

Table I sums up the conceptual relationships in local realism and macrorealism. It is worth mentioning that in principle there exist situations where no-signaling is violated although no sufficient number of setting is involved to construct a Bell inequality. This is the case when Bob has only one possible setting, say $b_{2}$, and his outcome $B$ reveals Alice's setting choice. Such a model violates local realism (1) and no-signaling (4), but no Bell inequality can be constructed with only two correlation functions $C_{a_{1} b_{2}}$ and $C_{a_{2} b_{2}}$. Similarly, if only two temporal correlations $C_{t_{1} t_{4}}$ and $C_{t_{3} t_{4}}$ are allowed to be measured, no Leggett-Garg inequality can be violated. However, a violation of macrorealism (5) and no-signaling in time (8) remains detectable.

To exemplify the usefulness of no-signaling in time, we consider a double slit experiment with large objects. Each object is emitted at the time $t_{0}$, passes a double slit at time $t_{1}$, and arrives at a detection screen at time $t_{2}$. As macrovariable we choose the lateral position variable denoted by $x$. We assume that the slit distance $d$ is large enough to qualify for the term "macroscopically distinct". Now three experiments are performed, each with many runs: I. Both slits are open. II. The left slit is blocked by a detector. Only objects passing the right slit will reach the detection screen at $t_{2}$. These are the ones which, according to a macrorealist, cannot be influenced by the measurement at $t_{1}$ at the other slit (ideal negative result measurements [3, 32]). III. Same as experiment II but with the right slit blocked. No-signaling in time predicts that the distribution $P_{\mathrm{I}}\left(x_{t_{2}}\right)$ found in experiment I must be the same as the weighted mixture $P_{\text {II\&III }}\left(x_{t_{2} \mid x_{t_{1}}}\right)$ of the single-slit distributions found in experiments II and III. Quantum mechanics, on the other hand, predicts interference between macroscopically distinct states, and thus an interference pattern for $P_{\mathrm{I}}\left(x_{t_{2}}\right)$, but no interference fringes for $P_{\mathrm{II} \& \mathrm{III}}\left(x_{t_{2} \mid x_{t_{1}}}\right)$. It is important to note that there seems to be no way to write down a violable Leggett-Garg inequality for the double-slit experiment.

For a Mach-Zehnder interferometer, a three-time LeggettGarg inequality of the Wigner form [33] $C_{t_{0} t_{1}}+C_{t_{1} t_{2}}-C_{t_{0} t_{2}} \leq$ 
1 can be employed. Before the first beam splitter (time $t_{0}$ ), inside the interferometer (time $t_{1}$ ), and after the second beam splitter (time $t_{2}$ ), there are always two possible paths $\left(Q_{t_{0}}, Q_{t_{1}}, Q_{t_{2}}= \pm 1\right)$. Depending on the four parameters-the initial probability distribution for $Q_{t_{0}}$, two reflectivities, and one phase shift in the interferometer-one can find regimes where the Leggett-Garg inequality is obeyed but, according to quantum mechanics, no-signaling in time is violated and others where the opposite is the case. For instance, if both beam splitters are balanced with reflectivities $\frac{1}{2}$, the LeggettGarg inequality becomes $-C_{t_{0} t_{2}} \leq 1$ and cannot be violated. No-signaling in time, on the other hand, demands $P\left(Q_{t_{2}}\right)=P\left(Q_{t_{2} \mid t_{1}}\right)$ and is violated by an intermediate measurement inside the interferometer at $t_{1}$ for all parameter choices which allow interference. In contrast, for an initial mixture $P\left(Q_{t_{0}}= \pm 1\right)=\frac{1}{2}$, no-signaling in time cannot be violated, while the Leggett-Garg inequality can be violated for a suitable choice of reflectivities and phase. (For more details see the Appendix.) This demonstrates that neither the violation of the 3-time Leggett-Garg inequality nor the violation of nosignaling in time is necessary for a violation of macrorealism.

Neither realism nor macrorealism per se can be tested on their own, which is why experimental tests have to be carefully designed to avoid loopholes. The three main loopholes in Bell experiments-locality, fair sampling, and freedom of setting choice-have all been closed individually [34-39]. In macrorealism, the non-invasiveness loophole should be closed by performing ideal negative result measurements [3. [32], which has been achieved already for microscopic systems [29]. Closing the fair sampling and freedom-of-choice loopholes will require high detection efficiency and statistical independence between the measured macroscopic object and the chosen measurement times just as in Bell tests.

Assume that one day a loophole-free experiment is performed which violates the Leggett-Garg inequality (or nosignaling in time) for macroscopic observables, thereby ruling out objective collapse theories [11-13]. The Bohmian interpretation of quantum mechanics [40] would still claim a well-defined position for every object at all times and allow for a description obeying macrorealism per se (as it would still provide a realistic description of a loophole-free Bell test). Ideal negative result measurements do not change the position macrovariables themselves, but they alter their subsequent time evolution due to an instant (non-local) change of the quantum wave function which serves as a guiding potential, thus violating the non-invasiveness condition. Bohmian mechanics is realistic, non-local, and no-signaling in the language of Bell, and it is macrorealistic per se, invasive, and signaling in time in the language of Leggett-Garg and the present work. Even if, as the authors, one does not adhere to this interpretation, this indicates a deeper connection between locality and non-invasive measurability beyond their formal analogy.

Conclusion. We have identified "no-signaling in time" as an alternative necessary condition for macrorealism which is different from the Leggett-Garg inequalities. Both conditions, no-signaling in time and the Leggett-Garg inequalities, are im- plied by macrorealism, but in general neither implies the other and neither violation is necessary for a violation of macrorealism. However, there are two main advantages of no-signaling in time, making it appealing for future experiments: 1 . While a Leggett-Garg test needs to involve at least three possible measurement times, no-signaling in time requires only two, allowing for tests in situations, where Leggett-Garg inequalities cannot be used at all. 2. As no-signaling in time can be violated by any non-vanishing interference term, it usually can be violated for a much wider parameter regime than the Leggett-Garg inequalities. Finally, one might argue that a violation of no-signaling in time is a direct violation of noninvasive measurability and that one is interested only in those situations where no-signaling in time is obeyed but a LeggettGarg inequality is violated. However, we note that a violation of no-signaling in time-just as a violation of the LeggettGarg inequality — can be achieved using ideal negative measurements. Violating no-signaling in time is thus no more a violation of non-invasive measurability (or of macrorealism per se) than a violation of the Leggett-Garg inequality itself. In summary, our work has shown that the step from a stage 2 experiment (showing quantum interference of macroscopically distinct states) to a stage 3 experiment (stage 2 and simultaneously ruling out macrorealism) does not require the complexity of the Leggett-Garg inequalities. It suffices to test the simpler criterion of no-signaling in time.

Note added in proof. Recently, a related work [41] has been submitted and published.

Acknowledgments. We acknowledge discussions with M. Aspelmeyer, J. I. Cirac, B. Dakić, O. Romero-Isart, and A. J. Leggett.

\section{Appendix}

Let us consider in more detail the example of a MachZehnder interferometer (see Fig. 1). Because there are always two possible paths before the first beam splitter (time $t_{0}$ ), inside the interferometer (time $t_{1}$ ), and after the second beam splitter (time $t_{2}$ ), the macrovariable $Q$ can always take one of two possible values: $Q_{t_{0}}, Q_{t_{1}}, Q_{t_{2}}= \pm 1$. The reflectivities of the first and second beam splitter are denoted by $R_{1}$ and $R_{2}$, and the phase shift in the lower arm is called $\varphi$. We choose an arbitrary initial distribution $P\left(Q_{t_{0}}=+1\right)=q$, $P\left(Q_{t_{0}}=-1\right)=1-q$ of incoming objects in a mixed quantum state $q|+1\rangle\langle+1|+(1-q)|-1\rangle\langle-1|$ with $|+1\rangle$ and $|-1\rangle$ corresponding to the macrovariable values +1 and -1 , respectively.

Quantum mechanics predicts the following temporal correlations $C_{t_{i} t_{j}}=\sum_{k, l= \pm 1} k l P\left(Q_{t_{i}}=k, Q_{t_{j}}=l\right)$ between times $t_{i}$ and $t_{j}$ :

$$
\begin{aligned}
& C_{t_{0} t_{1}}=1-2 R_{1}, \\
& C_{t_{1} t_{2}}=2 R_{2}-1, \\
& C_{t_{0} t_{2}}=-1+2 R_{1}+2 R_{2}-4 R_{1} R_{2}+4 \sqrt{R_{1} T_{1} R_{2} T_{2}} \cos \varphi,
\end{aligned}
$$

with the transmittances $T_{1}=1-R_{1}$ and $T_{2}=1-R_{2}$. The 


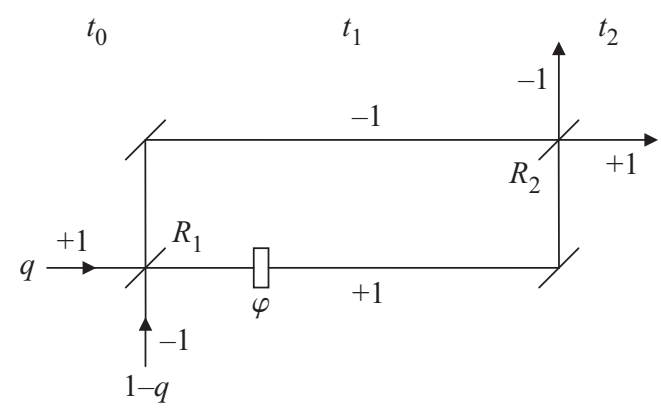

FIG. 1: Schematic of the Mach-Zehnder interferometer.

correlation functions are independent of $q$ because the first measurement always acts as a preparation for the second. The Leggett-Garg inequality $K \equiv C_{t_{0} t_{1}}+C_{t_{1} t_{2}}-C_{t_{0} t_{2}} \leq 1$ reads

$$
1-4 R_{1} T_{2}-4 \sqrt{R_{1} T_{1} R_{2} T_{2}} \cos \varphi \leq 1 .
$$

The maximum violation of $K=1.5$ is achieved for $R_{1}=\frac{1}{4}$, $R_{2}=\frac{3}{4}$, and $\varphi=\pi$. No violation is possible, e.g., if $R_{1}=$ $R_{2}=\frac{1}{2}$, because then $C_{t_{0} t_{1}}$ and $C_{t_{1} t_{2}}$ vanish and the remaining inequality $-C_{t_{0} t_{2}} \leq 1$ is always fulfilled.

No-signaling in time demands

$$
P\left(Q_{t_{2}}=+1\right)=\sum_{Q_{t_{1}= \pm 1}} P\left(Q_{t_{1}}, Q_{t_{2}}=+1\right) .
$$

The left hand side is the probability for outcome +1 at time $t_{2}$ without any prior measurements and is, according to quantum mechanics, given by $\frac{1}{2}+\frac{1}{2}(2 q-1) C_{02}$. The right hand side is the same probability but in the case of an intermediate measurement at time $t_{1}$ and is given by $\frac{1}{2}+(2 q-1)\left(-\frac{1}{2}+R_{1}+R_{2}-\right.$ $2 R_{1} R_{2}$ ). The difference between the left and the right hand side, which must vanish if no-signaling in time holds, reads

$$
2(2 q-1) \sqrt{R_{1} T_{1} R_{2} T_{2}} \cos \varphi=0 .
$$

This is violated whenever the parameters allow for interference, i.e., when neither of the reflectivities is 0 or 1 , the phase is unequal to $\frac{\pi}{2}$ and $q$ is unequal to $\frac{1}{2}$. The biggest violation (largest interference) is achieved for $R_{1}=R_{2}=\frac{1}{2}, q=0$ or 1 , and $\varphi=0$ or $\pi$.

For many parameter choices the Leggett-Garg inequality and no-signaling in time are both violated or both fulfilled. However, there are also parameter regimes (e.g., $R_{1}=\frac{1}{4}$, $R_{2}=\frac{3}{4}, \varphi=\pi$, and $q=\frac{1}{2}$ ) for which quantum mechanics violates the Leggett-Garg inequality while no-signaling in time is fulfilled, and others (e.g., $R_{1}=\frac{1}{2}, R_{2}=\frac{1}{2}, \varphi=\pi$, and $q=1$ ) for which no-signaling in time is violated while the Leggett-Garg inequality is satisfied. This demonstrates in general that-although both the Leggett-Garg inequality and nosignaling in time are a consequence of macrorealism-neither of these two criteria implies the other. Therefore, neither the violation of the Leggett-Garg inequality nor the violation of no-signaling in time is a necessary condition for a violation of macrorealism.
[1] J. S. Bell, Physics (New York) 1, 195 (1964).

[2] M. A. Nielsen and I. L. Chuang, Quantum Computation and Quantum Information (Cambridge University Press, 2000).

[3] A. J. Leggett and A. Garg, Phys. Rev. Lett. 54, 857 (1985).

[4] J. R. Friedman et al., Nature 406, 43 (2000).

[5] A. Palacios-Laloy et at., Nature Phys. 6, 442 (2010).

[6] M. Arndt et al., Nature 401, 680 (1999).

[7] S. Gerlich et al., Nature Comm. 2, 263 (2011).

[8] B. Julsgaard, A. Kozhekin, and E. S. Polzik, Nature 413, 400 (2001).

[9] O. Romero-Isart et al., Phys. Rev. Lett. 107, 020405 (2011).

[10] E. Schrödinger, Die Naturwissenschaften 48, 807 (1935).

[11] G. C. Ghirardi, A. Rimini, and T. Weber, Phys. Rev. D 34, 470 (1986).

[12] L. Diósi, Phys. Rev. A 40, 1165 (1989).

[13] R. Penrose, Phil. Trans. R. Soc. Lond. A 356, 1927 (1998).

[14] A. J. Leggett, J. Phys.: Cond. Mat. 14, R415 (2002).

[15] R. K. Clifton, in Symposium on the Foundations of Modern Physics 1990, P. Lahti and P. Mittelstaedt, eds. (World Scientific, Singapore, 1991).

[16] S. Foster and A. Elby, Found. Phys. 21, 773 (1991).

[17] F. Benatti, G. C. Ghirardi, and R. Grassi, Found. Phys. Lett. 7, 105 (1994).

[18] J. Kofler and Č. Brukner, Phys. Rev. Lett. 101, 090403 (2008).

[19] T. Fritz, New J. Phys. 12, 083055 (2010).

[20] J. F. Clauser and A. Shimony, Rep. Prog. Phys. 41, 1881 (1978).

[21] J. F. Clauser et al., Phys. Rev. Lett. 23, 880 (1969).

[22] S. Popescu and D. Rohrlich, Found. Phys. 24, 379 (1994).

[23] Č. Brukner et al., e-print arXiv:quant-ph/0402127 v1 (2004).

[24] J. Kofler and Č. Brukner, Phys. Rev. Lett. 99, 180403 (2007).

[25] J. Dressel et al., Phys. Rev. Lett. 106, 040402 (2011).

[26] M. E. Goggin et al., Proc. Natl. Acad. Sci. USA 108, 1256 (2011).

[27] A. Fedrizzi et al., Phys. Rev. Lett. 106, 200402 (2011).

[28] G. Waldherr et al., Phys. Rev. Lett. 107, 090401 (2011).

[29] G. C. Knee et al., Nature Comm. 3, 606 (2012).

[30] A. Peres, Quantum Theory: Concepts and Methods (Kluwer Academic, Dordrecht, 1995).

[31] B. Dakić, T. Paterek, and Č. Brukner, in preparation.

[32] A. J. Leggett, Found. Phys. 18, 939 (1988).

[33] E. P. Wigner, Am. J. Phys. 38, 1005 (1970).

[34] A. Aspect, J. Dalibard, and G. Roger, Phys. Rev. Lett. 49, 1804 (1982).

[35] G. Weihs et al., Phys. Rev. Lett. 81, 5039 (1998).

[36] M. A. Rowe et al., Nature 409, 791 (2001).

[37] M. Ansmann et al., Nature 461, 504 (2009).

[38] T. Scheidl et al., Proc. Natl. Acad. Sci. USA 107, 19708 (2010).

[39] M. Giustina et al., arXiv:1212.0533 Nature advance online publication 14 April 2013.

[40] D. Bohm, Phys. Rev. 85, 166 (1952).

[41] C.-M. Li et al., Sci. Rep. 2, 885 (2012).

[42] Equivalently to eq. (1), local realism guarantees the existence of a joint probability distribution $P\left(A_{a_{1}}, A_{a_{2}}, \ldots, B_{b_{1}}, B_{b_{2}}, \ldots\right)$ for the results of all possible (mutually exclusive) measurement results. Here, $A_{a_{1}}$ denotes Alice's outcome for setting $a_{1}$ and so on. In particular, due to locality, there is no need to distinguish local outcomes for different distant settings.

[43] For uncountable outcomes, integrals instead of sums are used.

[44] Equivalently to eq. (5), macrorealism guarantees the existence of a joint probability distribution $P\left(Q_{t_{1}}, Q_{t_{2}}, \ldots, Q_{t_{1}}^{\prime}, Q_{t_{2}}^{\prime}, \ldots\right)$ for all macrovariables at all times. In particular, due to non-invasive 
measurability, there is no need to distinguish later outcomes for different possible actions at earlier times.

[45] Note the two different levels of 'deterministic versus stochastic': One level asks whether the complete description at a later time follows deterministically from the one at an earlier time. The other one asks whether an individual outcome is determined by the complete description at the time of measurement. A local realist is free to answer both questions negatively. As a macrorealist, one is also free regarding the first question but one has to answer the second one positively. (If you limit yourself to
Newtonian physics, you also need to answer the first question positively.)

[46] The overlap between the two measured probability distributions is, for example, quantified by $\kappa \equiv \sum_{B} \sqrt{P\left(B_{t_{B}}\right) P\left(B_{t_{B} \mid t_{A}}\right)} \in[0,1]$. A violation of (8) can be stated if identical distributions $(\kappa=1)$ are ruled out in a statistically significant way. The issues of finite data sets, measurement inaccuracies, and statistical significance also arise in tests of Bell or Leggett-Garg inequalities. 